\title{
Cymatics Inspired Self-Cleaning Mechanism for Solar Panels
}

\author{
Edwin Babu', Sumith Yesudasan ${ }^{2 *}$ and Sibi Chacko ${ }^{1}$ \\ ${ }^{1}$ School of Engineering and Physical Sciences, Heriot-Watt University, Dubai, UAE \\ ${ }^{2}$ Department of Mechanical Engineering, University of Jamestown, Jamestown, ND, USA
}

\begin{abstract}
Author emails:
Edwin Babu: ekb1@hw.ac.uk

Sibi Chacko: c.sibi@hw.ac.uk

*Sumith Yesudasan: sumith.yesudasan@uj.edu
\end{abstract}

\begin{abstract}
The Photovoltaic modules are usually installed on the ground which exposes it to surface deposition of foreign particles. In the Middle East and North Africa region, the primary culprit is dust and sand. They form an insulating and opaque layer on the surface of the glass, which obstructs its heat transfer and optical properties, thereby reducing the overall yield efficiency of the solar panel. Cleaning of this layer is critical to the operation of the solar panel and often requires great effort and energy on a large-scale solar array. In this paper, we propose a novel self-cleaning mechanism for solar panels, with an understanding of the structural integrity of the Photovoltaic laminate and application of external mechanical vibration. By applying an external source of vibration, the solar panels vibrate, excites its fundamental frequencies and cleans by its own. The method is analyzed using finite element analysis method and tested using experiments. Our simulation results based on IEC 61215 show that the maximum principal stress and deformation in the critical layers is within limits. Our experimental results prove the proposed theory is feasible and can be extended to large scale solar arrays. Our proposed method is retrofittable and could save money, energy and effort in cleaning the solar arrays, which can replace current techniques.
\end{abstract}

Keywords: Solar Panel, Laminate, Vibrations, Self-Cleaning, Cymatics

\section{Introduction}

Over the years, the demand for energy has increased, and the implementation of solar power systems is being investigated as it is potentially capable of zero-emissions during electricity production while being environmentally friendly and economical to the general masses. The fundamental unit of the solar module is the doped silicon, provides an overall efficiency of the generated electricity from the module to be 15-20\% [1]. However, the potential electricity yield can be enhanced by optimizing specific parameters such as the angle of orientation, exposure area, sun-tracking devices etc. This is to be taken into consideration as the practicality of solar modules drops due to the deposition of particles, unanticipated droppings and sedimentation on the surface of the module which brings down the overall efficiency of the system.

With the increase in the application of personal and grid-based Photovoltaic (PV) module systems alongside expanding solar power plantations in the regions of low-altitude deserts such as the Middle East where the maximum sunlight can be harvested comes along with a rising issue of dust soiling of the panels which forms in the microscale region $(50-150 \mu \mathrm{m})$. The effect of wind coupled with gravity promotes dust settlement on the surface as low-speed winds tend to accumulate these particles on the panel (initial layer of dust attracts further particles which aggregate over time) while on the contrast, high-speed winds cause the layer to disperse [2]. These dust particles hinder the process for electricity generation by blocking the photons from the sunlight and when viewed from a large-scale perspective in a solar plant can cause a major dip in its efficiency output if not dealt with appropriately. Therefore, it is essential for a reliable and economical cleaning system to be implemented. Initially, it was found that the module efficiency is dropped drastically due to an obstructed panel surface, and this is to be avoided by cleaning the panel regularly every 2-3 weeks for rated efficiency [3]. Multiple methods were researched and established by scientists for tackling this issue.

Currently, there exist three main approaches based on location and size of the solar grid of interest that is Electrostatic, Nano-film/coating, Mechanical method for panel cleaning [4]. The electrostatic methods were found to be useful in repelling dust as they carry a charge which is opposed by electrodes attached to the glass surface, making the particles bounce off in succession cleaning the surface. This method is useful for small-scale panels as they are still under research from the past decade, and the cost of electrodes along with their connecting wiring setup is a limiting factor [5]. Nanofilm is viable for large solar grids due to its ease of application by spray coating the glass surface and hydrophobic/self-cleaning properties as it repels dust, pollen etc. forming on the panel which is then washed or carried away from rain or mild winds respectively. If not appropriately applied, the varying thickness of the coating could potentially affect the transmissivity reducing the yield efficiency; subsequently reapplying is costly per liter of the nanofilm solution [6]. Mechanical based cleaning systems operate with machinery (moving equipment's) and labor (manual scrubbing using telescopic brushes that could potentially cause panel abrasion) which relies on a considerable amount of power input similar to the electricity requirements for the working of electrostatic methods [3]. While each of these individual methods has their respective merits 
Cymatics Inspired Self Cleaning Mechanism for Solar Panels and shortcomings, there is always room for improvement by being able to establish a system for cleaning in all aspects while consuming minimal power and maintaining the structural integrity of the solar module.

In this work, we propose a self-cleaning method inspired from the concept of Cymatics [7], in which at certain frequencies physical patterns of the mode shapes are observed on the surface of the membrane that is being exposed to vibrations. We investigate the (i) structural integrity of the laminate from the frequency response of vibrations and (ii) Mechanical stresses within the critical layers of the laminate (Glass and Silicon wafer) using Finite Element Analysis (FEA) and (iii) Experimental testing of the proposed method.

\section{Theoretical Aspect}

\subsection{Unbalanced force and Wave equation}

By considering the laminate of the solar module as an effective rectangular slab/sheet, the laminate is to be subjected to an externally applied force to create vibrations within the system. This is done by attaching a DC motor directly to the back-sheet of the panel which is supported on four edges to emulate a system of mass ' $M$ ' being excited by an unbalanced mass to induce vibrations. The system is then represented by Eq. 1 in terms of forces according to Newtons $2^{\text {nd }}$ Law with ' $h$ ' as the eccentricity of the attached mass ' $m$ ' to the motor and ' $m h \omega^{2} \sin (\omega t)$ ' as the external force applied with ' $\omega$ ' as the excitation frequency.

$M \ddot{y}+c \dot{y}+k y=m h \omega^{2} \sin (\omega t)$

The response equation of the system is then obtained from the vector diagram projected by Equation 1 from which the natural frequency ' $\omega_{n}$ ' is calculated from the frequency ratio ' $\mathrm{n}$ ' $\left(n=\omega / \omega_{n}\right)$. The damping ratio of the system is ' $\zeta$ ' which is calculated from the logarithmic decrement that is the ratio of amplitude for the system to reach equilibrium when excited with an external force.

$\frac{M Y_{O}}{m h}=\frac{n^{2}}{\sqrt{\left(1-n^{2}\right)+(2 \zeta n)^{2}}}$

Therefore, the excitation from the eccentric motor causes vertical displacement within the laminate (rectangular sheet) and is described as a 2D differential wave-equation [8] [9] of the form

$\mathrm{D} \nabla^{2} \nabla^{2} v(x, z, t)=f(x, z, t)-\rho t \frac{\partial^{2} v}{\partial t^{2}}(x, z, t)$

Here ' $\rho$ ' and ' $t$ ' are the respective density and thickness of the material with the deflection ' $v$ ' and forced response $f(x, z, t)$ being co-dependent to space and time.

\subsection{Experimental design selection}

The setup was arranged to be constrained firmly and allow vertical vibrations throughout the laminate. By varying the voltage from the DC power supply, the rpm is controlled for the RS-540 SH motor (20000rpm at no load).

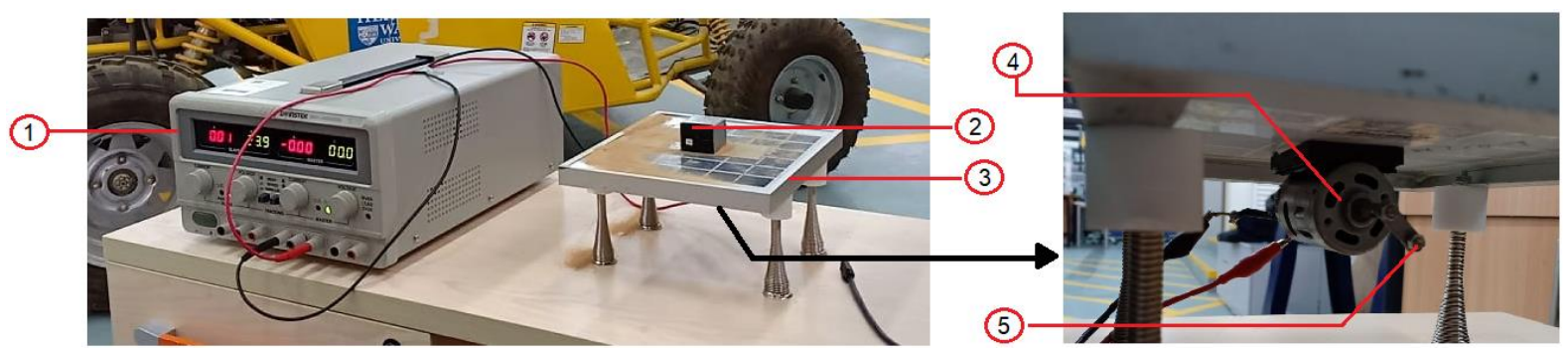

Figure 1: (1) DC Power Supply, (2) Wireless Accelerometer, (3) Solar Panel, (4) DC Motor, (5) Eccentricity mass.

The response to excitation ' $\mathrm{Y}_{\mathrm{o}}$ ' in $\mathrm{mm}$ is measured using the SQI wireless accelerometer attached to the surface, which is set to take 510 samples per second. Data is collected as the amplitude of vibration vertically for 8 seconds by varying the voltage from $1 \mathrm{~V}-4.2 \mathrm{~V}$ before stalling the motor. This data is then run through a Discrete Fourier Transform (DFT) code on MATLAB R2016a [10] to convert the data into the frequency domain clearing out the noise and filtering the signal from which the excitation frequency is then obtained.

\section{Finite Element Analysis (FEA) Simulation}

\subsection{Implementation of the FEA model}


The first model was designed as a foundation for validating the FEA model to the experimental setup. According to the literature [11], a FEA solar module was emulated from the author Jinzhi Dong et al. in which the respective author carried out a static and dynamic analysis of the IEC 61215 standardization test [12]. It stated that the PV module should be able to withstand wind/snow loads which are at $2400 \mathrm{~Pa}$ and $5400 \mathrm{~Pa}$ respectively. From the author's description, the model was simulated for the 4-edge mounting configuration, which is vastly used for fastening the PV module securely while maintaining high yield efficiency [11]. The total deformation and the maximum principal stress for the mounting configuration selected as obtained by the author were $3.85 \mathrm{~mm}$ and 61.18MPa respectively [11]. This was used to verify the simulated model on ANSYS which resulted as $3.874 \mathrm{~mm}$ and $61.267 \mathrm{MPa}$ with the accuracy error of $0.6 \%$ and $0.14 \%$ for the total deformation and maximum principal stress obtained respectively which is well within the range of modelling error.

\subsection{Modeling Approach}

The primary part to be modelled being the laminate consists of 5 layers with the ethylene-vinyl acetate (EVA) sandwiching the Si cell, which has a Tedlar back-sheet and a tempered glass on top. These layers come together in the laminator during the lamination process, which occurs at a temperature of $150^{\circ} \mathrm{C}$ curing the EVA and bonding with the respective layers to form a single unit [12]. The EVA generally acts as a shock-absorbent (encapsulant) in cases of random vibrations or mishandling of the module, protecting the Silicon cell within. This laminate is then attached to the Aluminum 6065 frame completing the basic solar module.
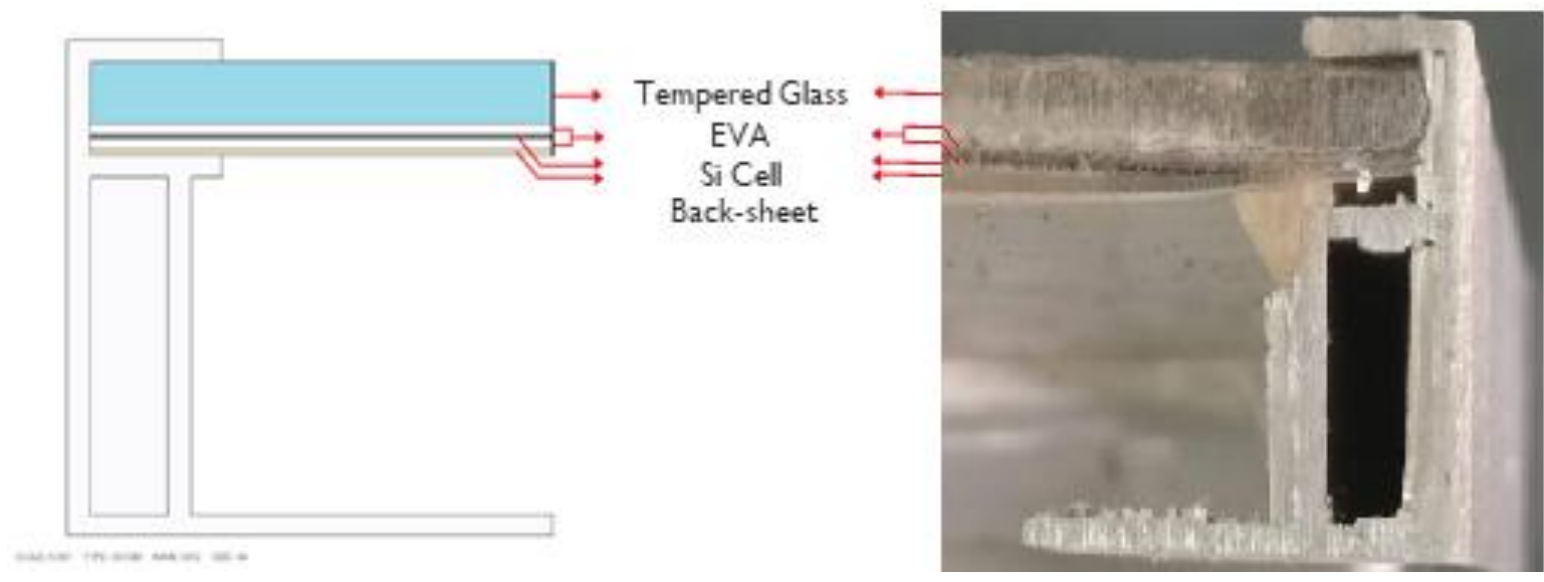

Figure 2: Creo Drawing of the Laminate in the frame (Left) PV module sectioned view from the lab (Right)

To optimize the computational cost for the simulation purpose, the frame along with the busbars is not modelled with the laminate as the excitation frequency is directly transmitted to the module and its corresponding response to this force is to be investigated. The weight distribution and its respective effect on the laminate are instead applied via the boundary condition. The model was initially made on Creo Parametric 3.0 with the dimensions of the current panel as specified by the manufacturer $(185 \mathrm{~mm} \times 220 \mathrm{~mm} \times 17 \mathrm{~mm})$. This was then imported to Ansys, where the contact regions were refined, and the experimental environment was replicated.

Table 1: Structural material properties

\begin{tabular}{|c|c|c|c|c|}
\hline Material & Density & Thickness & Poisson Ratio & Youngs Modulus \\
\hline & $\mathrm{kg} / \mathrm{m}^{3}$ & $\mu \mathrm{m}$ & & $\mathrm{GPa}$ \\
\hline Tempered Glass & 2270 & 3430 & 0.23 & 70 \\
\hline EVA & 1030 & 500 & 0.33 & 0.0677 \\
\hline Si Cell & 2329 & 250 & 0.28 & 112.4 \\
\hline Back-sheet & 2520 & 320 & 0.29 & 3.5 \\
\hline
\end{tabular}

\subsection{Simulating the setup}

The model is simulated through consecutive stages on Ansys with the result of every step being transferred to the subsequent step (Static Structural $\rightarrow$ Modal Analyses $\rightarrow$ Harmonic Response) to emulate the real-life testing. In the first step, the respective boundary conditions are applied, which is similar to the laminate being constrained along its four edges with the gravitational force acting on the Y-axis. The weight of the motor and supports are considered as well. The result from the former step is then evaluated to obtain the mode shapes of the system which returns the natural frequencies of the setup. During the Harmonic response step, the unbalanced force is applied to the laminate, which provides the frequency response graph against the amplitude for a specific excitation frequency. 


\subsubsection{Mesh Convergence}

For the mesh, the laminate is the critical part to be evaluated, which contains the glass layer over the encapsulant. Therefore, the mesh is established to be dense throughout the surface area since the stresses and potential deformation needs to be investigated. Due to the symmetry of the model, the laminate is meshed by no. of divisions over the surface and segmented into smaller layers through the thickness, as seen in Figure 3. The layers are partitioned into 8,4,2,2 divisions for Glass, EVA (each), Cell, Back-sheet respectively.

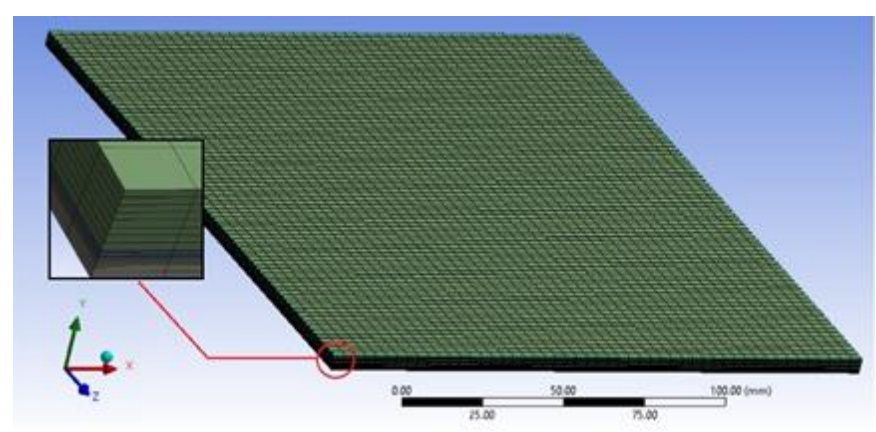

Figure 3: Mesh analysis and orientation on ANSYS

The mesh over the surface area was split into the $\mathrm{z}$ and $\mathrm{x}$-axis with a ratio of $3 / 2$ with the $\mathrm{z}$-axis taking the longer side as seen in Figure 3. To determine the ratio of divisions, the convergence was calculated for the Total deformation that the laminate experiences due to the mounting boundary conditions exposed to the gravitational force (Static structural step).

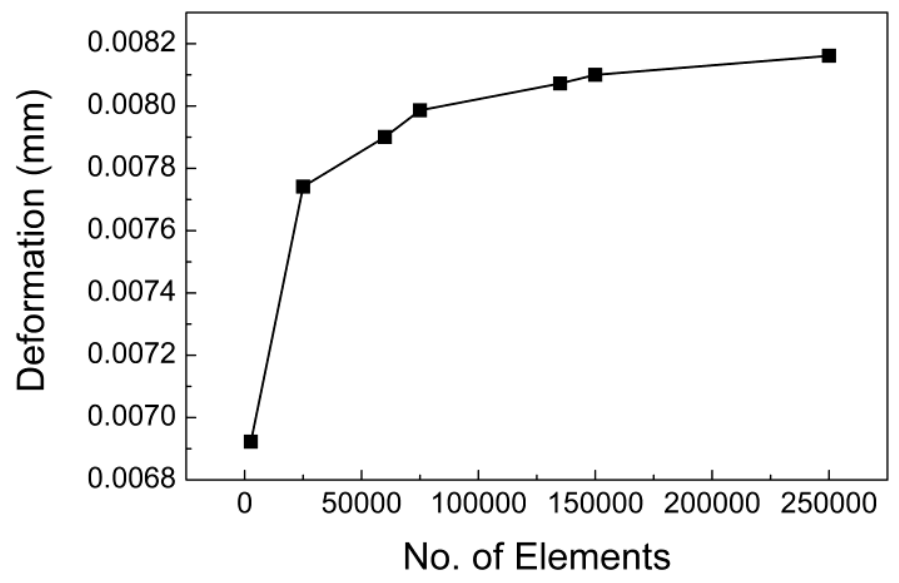

Figure 4: Convergence evaluation criteria

As the ratio was increased the no. of elements begins to exponentiate, this could potentially increase the computational time for obtaining the results, and therefore convergence is required. Multiple mesh configurations with varying no. of elements were simulated and recorded as seen in Figure 4. It was observed that the trend starts to asymptote at 75000 elements at which the total deformation was recorded to be $0.007986 \mathrm{~mm}$. The simulation was tested further for three more sets of data up to the allowed limit of the software for the current model, which was at 250000 elements and $0.00816 \mathrm{~mm}$ deformation respectively. The percentage error was calculated to be $2.2 \%$ at the point of asymptote, which therefore led to the conclusion of using the mesh configuration at 75000 elements since the error percentage is within the allowable limit.

\section{Results and Discussion}

\subsection{Finite Element Analysis step evaluation}

\subsubsection{Modal analyses and Mode shapes}

By identifying the mode shapes of the system, the respective resonant frequencies at which the structure deflects from vibrations is investigated. Primarily, there are two forms of vibrations which are the forced and the resonant vibrations, the former being a result of external excitation such as impulse, loading forces, unbalances etc. while the resonant vibration is occurring when the respective N-DOF within the structures vibrates at its corresponding modal shape. Furthermore, there exist two types of modes which is the rigid and flexible body modes. The rigid body mode branches out to 3 translational and 3 rotational modes respectively, while the flexible body modes can be variant depending on the constraints and external factors acting on the structure. It is beneficial to understand 
Cymatics Inspired Self Cleaning Mechanism for Solar Panels

that the mode shape is reliant on the dimensions of the laminate $(\mathrm{x}, \mathrm{z})$ and can have varying frequencies for a specific mode shape when the dimensions are changed.

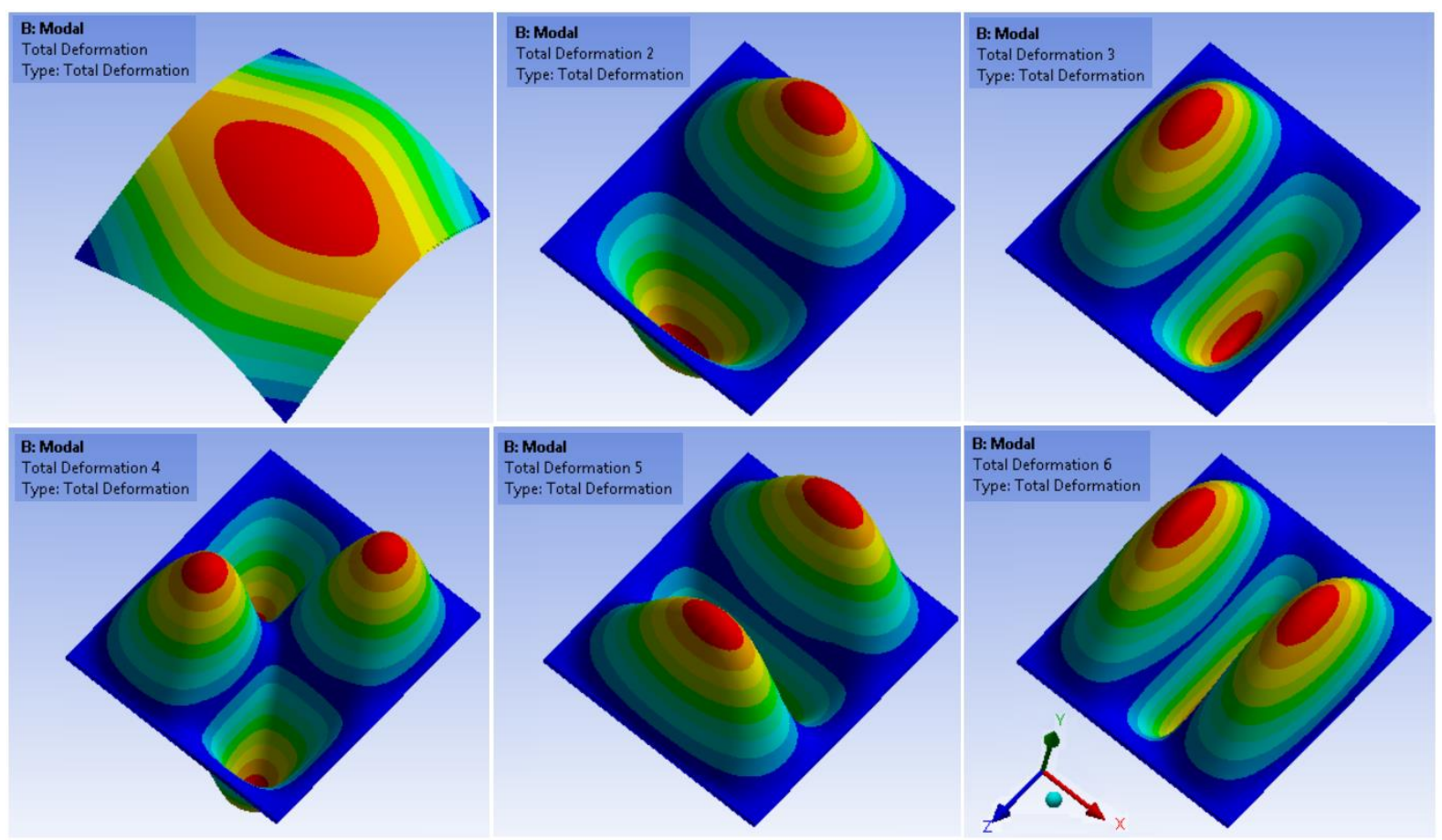

Figure 5: Simulation of the first six natural frequencies and mode shapes of the laminate

The mode shapes obtained in Figure 5 are similar to the standing wave patterns for a rectangular membrane fixed along the edges [13] which is of the form

$\varphi_{a, b}(x, z)=\sin \left(\frac{a \pi}{L_{x}} x\right) \sin \left(\frac{b \pi}{L_{z}} z\right)$

Where $L_{x} L_{z}$ are the dimensions of the laminate and the subscripts $(\mathrm{a}, \mathrm{b})$ is known as the mode shape identifiers which specifies the number of anti-nodes in the corresponding $\mathrm{x}$ and $\mathrm{z}$ regions respectively.

\subsubsection{Harmonic response of the laminate}

After the mode shapes and natural frequencies of the system setup was identified, the harmonic excitation was applied to the back-sheet of the laminate as a force of $m h \omega^{2}$ where ' $\omega$ ' is the operating frequency of the motor. The frequency response curve was obtained from Ansys in the $\mathrm{Y}$-axis direction, which gave the amplitude of vibration for varying excitation frequencies of which was compared to the experimental testing.

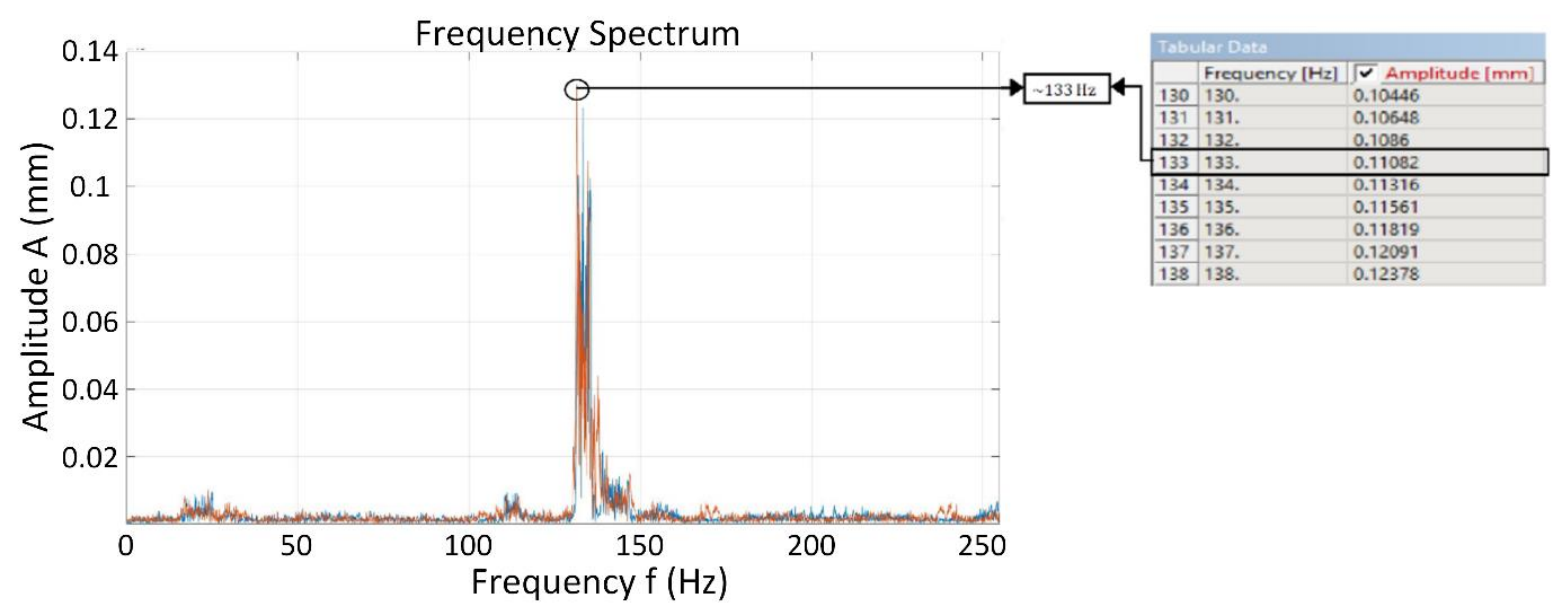

Figure 6: Model Validation of experimental (Left) to FEA modelling (Right) for the amplitude of vibrations

As mentioned in Section 2.2, the test was run multiple times at a specific rpm to obtain a clear amplitude of vibration of the laminate as seen in Figure 6 (Left) using the recorded data from the wireless accelerometer which is processed through the MATLAB code for Discrete Fourier Transform. It is to be noted that the selected frequency was within the limits of the motor load capacity. The highest peak obtained is at the operating frequency 
Cymatics Inspired Self Cleaning Mechanism for Solar Panels

Babu and et al.

with the corresponding amplitude of vibration to be $0.122 \mathrm{~mm}$ around $\sim 133 \mathrm{~Hz}$. This data is compared with the FEA simulation results in the tabular form, which was found to be $0.11082 \mathrm{~mm}$ at $133 \mathrm{~Hz}$. The accuracy of the responses is within the range of experimental error of tolerance $9.16 \%$

\subsection{Experimental testing evaluation}

\subsubsection{Source of contamination}

Depending on the location and size of the PV module, the effect of dust contamination coupled with a number of external factors aggregates to the accumulation of the deposited particles on the surface of the panel. According to a study conducted in Cairo for an array of PV cells, the author found that the efficiency of the panels dropped by $25 \%$ in the first two months from soiling and an overall of $40 \%$ across 12 months [14]. A medical geology study found the average particle size to range from $\sim 40-150 \mu \mathrm{m}$ by sampling the dust from various site locations within the Middle East [15]. Another study conducted in Doha tested and obtained the dust accumulation rate within the Middle East and North Africa (MENA) region to be $0.3 \mathrm{~g} / \mathrm{m}^{2}$ a day [16]. For the experimental analyses, two types of particle contaminants were used for simulating the soiling layer. The first test was conducted with the coarse sand particles (Diameter ranging $<300 \mu \mathrm{m}$ ) which tends to be airborne from sandstorms and heavy winds subsequently settling on the surface post-wind (Figure 7-Above). For the second test, dust was collected in a petri dish from an open space for four days which had a varying particle diameter of less than 50 $\mu \mathrm{m}$ (Figure 7-Below).

\subsubsection{Evaluation of vibrational cleaning}

The primary parameter that affects the cleaning process is the amplitude of vibrations. Following Section 4.1, as the rotor from the eccentric mass DC motor reaches the rotational speed of the first natural frequency, large amplitude of vibrations is induced on the panel. The intensity of the amplitude is controlled by the damping ratio of the system, which is the ability of the structure to dissipate energy caused by vibrations. For the experimental procedure, the logarithmic decrement ' $\delta$ ' is found by forcing an impact on the panel and recording the decaying curve to equilibrium from which the damping ratio is calculated. The speed at which the operating frequency matches the natural frequency is known as the critical speed, and the system is considered to be in the resonance of the first natural frequency. As the operating frequency is further increased, the amplitude of vibrations decreases gradually until the second natural frequency, where a rise in amplitude is observed. It is important to understand the mode shapes at the respective natural frequencies seen in Figure 5, as the concept of cleaning is based on the difference in amplitude within the surface.

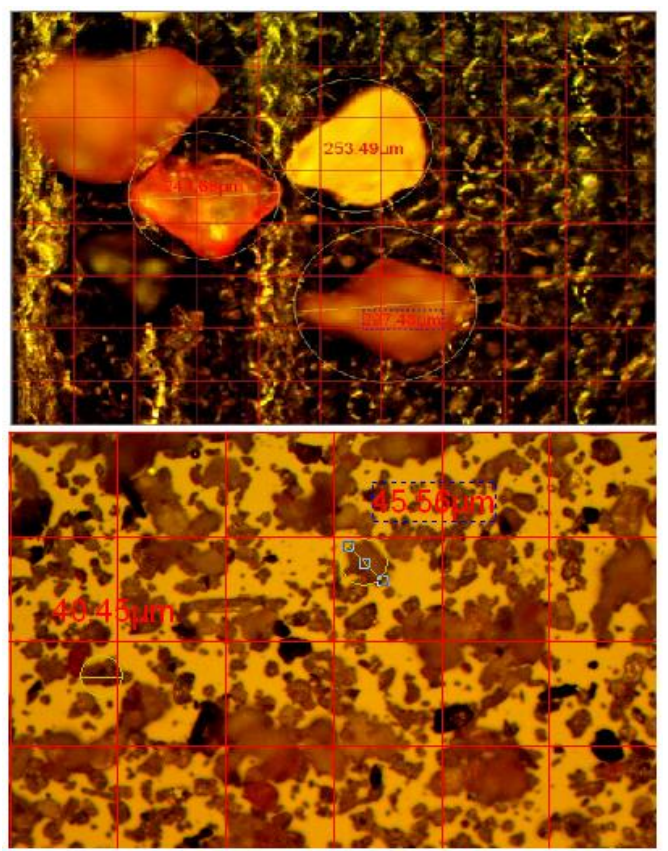

Figure 7: Sand and Dust samples for the experiment measured in lab

When the operating frequency approaches the first natural frequency, the amplitude of vibrations takes the form of the first mode shape which from the FEA is $174.95 \mathrm{~Hz}$. As the mode shape pattern begins to form, there is a point at which maximum vibrations develop on the surface known as the Anti-node and the counterpart of this being the Node where 0 amplitude of vibrations occur. Following this principle, the deposited particles (sand and dust) traverse from the anti-node to the node of the mode shape approaching the respective natural frequency as seen in Figure 8 where the operating frequency is reaching the $1^{\text {st }}$ natural frequency and follows the 
Cymatics Inspired Self Cleaning Mechanism for Solar Panels

vibrational pattern observed from the FEA for mode no. 1 where the amplitude of vibration is the maximum at the center of the panel while the nodal region exists at the corners.

\subsubsection{Effectiveness of cleaning}

To understand the overall cleaning potential using vibrations, the knowledge of adhesion of particles to a substrate is important. Primarily, the dominant forces of nature that determine the interaction between the particle and substrate depends on the size of the particle and the respective distance of separation amongst them. The adhesion of particles arises from the existence of attractive forces between the particle-substrate coupled with the mechanical response to this adhesion, that is the stress caused due to the deformation which determines the intensity of the bonded particle to the surface [17].

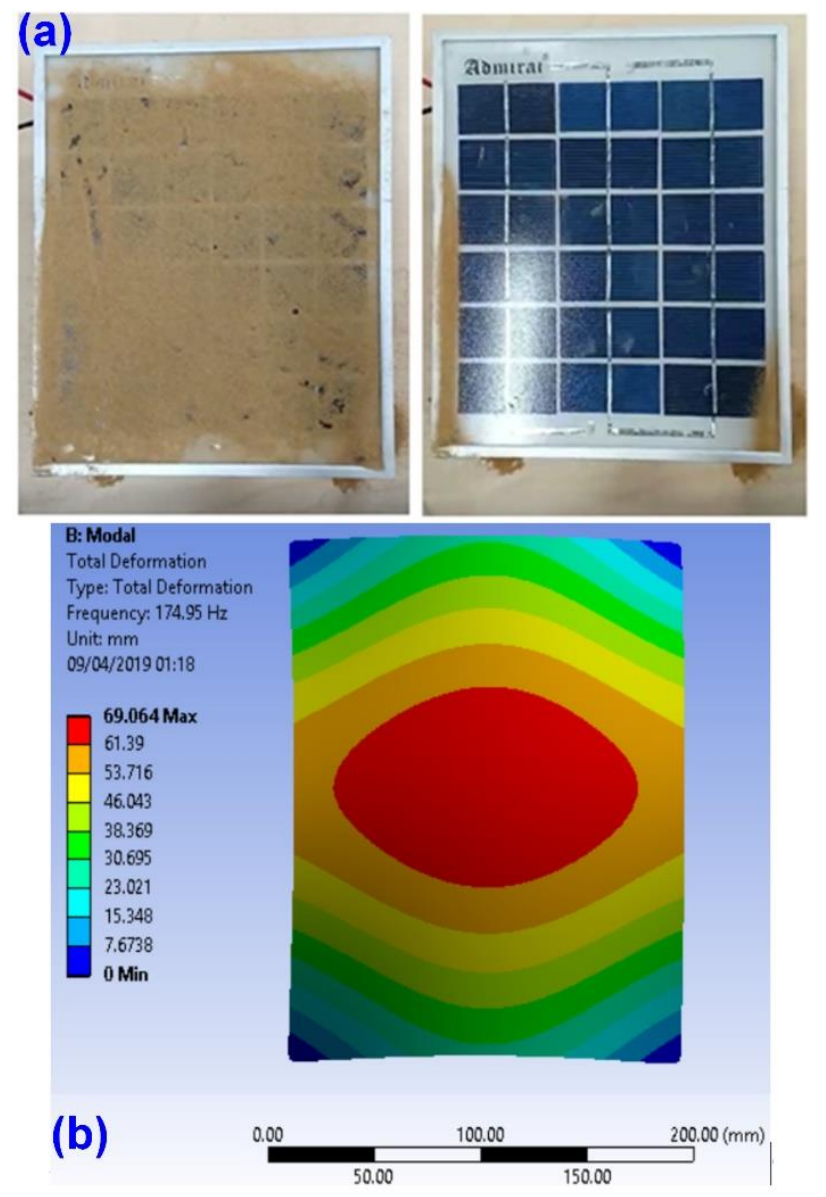

Figure 8: (a) Demonstration of vibrational cleaning before (left) and after (right). (b) The amplitude of vibration from the FEA model approaching first natural frequency

For the two tests conducted with varying particle diameter, it was observed that the cleaning efficiency for the sand $(\sim 300 \mu \mathrm{m})$ deposited layer was distinctively higher as seen in Figure 8 compared to the dust $(\sim 45$ $\mu \mathrm{m})$. As the operating frequency approached the $1^{\text {st }}$ natural frequency, any overlay of the dust thickness was reduced, leaving a fine layer of dust on the surface. This is explained in the literature [4] [17] as the Van der Waals forces between the particles of the dust can be excited from low frequencies but due to the large surface area of the substrate and reduced particle size $(<45 \mu \mathrm{m})$, the electrostatic forces of attraction are relatively high for the particle-substrate that causes the dust to adhere to the surface. As for the sand particles, the gravitational force $F_{g}=1.33 \pi R^{3} \rho g$ becomes a relevant factor, since the radius of the particle is dependent by a factor of 3 . Therefore, the force required to excite the particles for radius $>250 \mu \mathrm{m}$ increases exponentially which is a limitation for the size of the particles that can be potentially excited from the surface. The effect of adhesion forces of the particle-substrate is an important factor, and the removal of the particle is generally considered in terms of energy transfer rather than force equilibrium which makes it a quantitative problem since the particles of interest vary within a few microns, the qualitative analysis would be challenging due to the requirement of extensive knowledge in the field of particle adhesion and the respective equipment for testing.

\subsubsection{Stresses within the critical layers}

It is necessary to find the limit to which laminate can be exposed to forcing vibrations for avoiding failure and to preserve the lifetime of the PV module. An experimental study [18] conducted on a polycrystalline module for continuous cyclic bending which was done to further investigate the effect on the panels derived from the IEC 
Cymatics Inspired Self Cleaning Mechanism for Solar Panels

Babu and et al.

61215 standards for snow and wind loading. From the experiment results, it was established that the maximum tensile strength experienced by the Si cell was $\sim 300 \mathrm{MPa}$. This information confirms that the probability of the Silicon layer failing from brittle fracture is $<1 \%$ as the fracture strength of silicon is $\sim 1 \mathrm{GPa}$ [19]. Therefore, the critical crack propagation (brittle fracture) is avoided leaving the only potential failure which is the crack growth within the silicon due to fatigue at the maximum tensile stress [18]. The tempered glass layer which consists of the majority thickness of the laminate is also known as the safety glass due to its spontaneous fracture nature at which point it shatters to multiple small pieces; it has a tensile strength of 850-1000 MPa [20]. The overall deformation experienced by the laminate is $0.1636 \mathrm{~mm}$ at the excitation frequency of $133 \mathrm{~Hz}$ obtained from the FEA model.

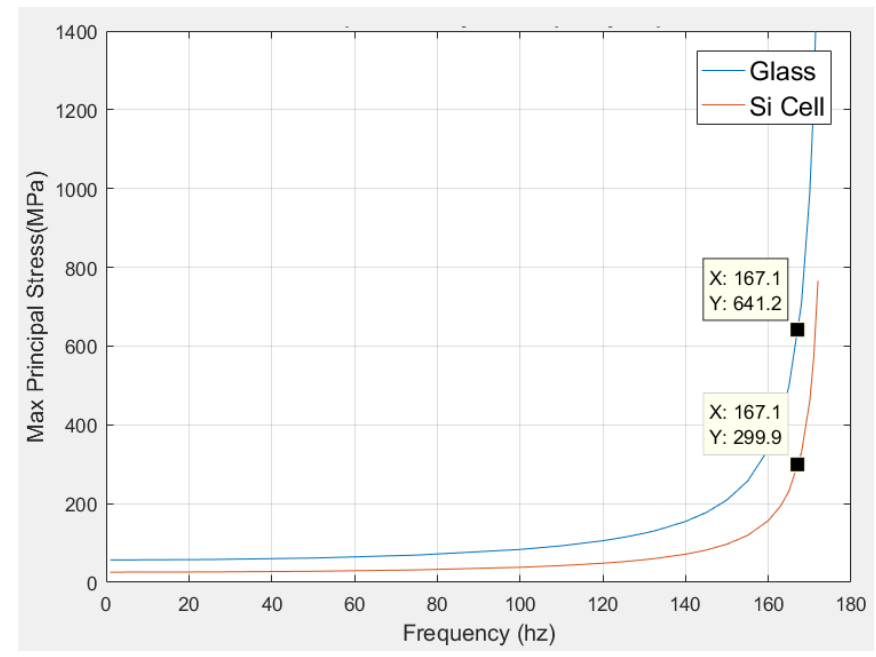

Figure 9: Maximum Principal Stress for varying excitation frequency. The silicon and glass layers are within the region of safety for the excitation frequency of $133 \mathrm{~Hz}$

\section{Conclusion}

The concept of cleaning solar panels using mechanical vibrations is investigated using computer simulations and using experimentation. The experiment was conducted by vibrating the panel near its natural frequencies, which will move the dust particles traverse from the anti-node to the node following the respective mode shapes. This effectively cleaned the surface of the solar panel. To check the structural integrity of the panel, whether it can withstand the vibrations without causing laminate damage, a FEA was performed. The FEA results show a maximum deformation of the laminate as $0.525 \mathrm{~mm}$ after which fatigue (sub-critical) of the silicon layer begins to occur at which the cell experiences maximum principal stress of $300 \mathrm{MPa}$. Our studies find that without damaging the structure of the solar panels, we can effectively utilize the concept of vibration-induced self-cleaning of solar panels.

\section{Acknowledgment}

We like to acknowledge Heriot-Watt University, Dubai for providing the research facilities \& grounds for testing and for funding the project.

\section{Conflict of Interest}

The authors declare no conflict of interest in preparing this article

\section{References}

[1] D. Deb and N. L. Brahmbhatt, "Review of yield increase of solar panels through soiling prevention, and a proposed water-free automated cleaning solution," Renewable and Sustainable Energy Reviews, vol. 82, pp. 3306-3313, 2018.

[2] M. Mani and R. Pillai, "Impact of dust on solar photovoltaic (PV) performance: Research status, challenges and recommendations," Renewable and sustainable energy reviews, vol. 14, no. 9, pp. 31243131, 2010.

[3] A. Syafiq, A. Pandey, N. Adzman, and N. A. Rahim, "Advances in approaches and methods for selfcleaning of solar photovoltaic panels," Solar Energy, vol. 162, pp. 597-619, 2018.

[4] E. Y.-T. Chen, L. Ma, Y. Yue, B. Guo, and H. Liang, "Measurement of dust sweeping force for cleaning solar panels," Solar Energy Materials and Solar Cells, vol. 179, pp. 247-253, 2018. 
Cymatics Inspired Self Cleaning Mechanism for Solar Panels

[5] H. Kawamoto and T. Shibata, "Electrostatic cleaning system for removal of sand from solar panels," Journal of Electrostatics, vol. 73, pp. 65-70, 2015.

[6] A. Gholami, A. A. Alemrajabi, and A. Saboonchi, "Experimental study of self-cleaning property of titanium dioxide and nanospray coatings in solar applications," Solar Energy, vol. 157, pp. 559-565, 2017.

[7] H. Jenny, "Cymatics: A Study of Wave Phenomena \& Vibration, 2001," Compiled Edition, original publication dates 1967, vol. 1, 1974.

[8] A. R. Pouladkhan, J. Emadi, M. Safamehr, H. Habibolahiyan, and others, "The vibration of thin plates by using modal analysis," World academy of science, Engineering and Technology, vol. 59, pp. 2880-2885, 2011.

[9] A. I. Abdul-Latif and J. Diaz, "Dirichlet, Neumann, and mixed boundary value problems for the wave equation uxx - uyy= 0 for a rectangle," Applicable Analysis, vol. 1, no. 1, pp. 1-12, 1971.

[10] C. Solomon and T. Breckon, Fundamentals of Digital Image Processing: A practical approach with examples in Matlab. John Wiley \& Sons, 2011.

[11] J. Dong, H. Yang, X. Lu, H. Zhang, and J. Peng, "Comparative study on static and dynamic analyses of an ultra-thin double-glazing PV module based on FEM," Energy Procedia, vol. 75, pp. 343-348, 2015.

[12] Y. Lee and A. A. Tay, "Stress analysis of silicon wafer-based photovoltaic modules under IEC 61215 mechanical load test," Energy Procedia, vol. 33, pp. 265-271, 2013.

[13] S. Kang and J. Lee, "Free vibration analysis of arbitrarily shaped plates with clamped edges using wave-type functions," Journal of Sound and Vibration, vol. 242, no. 1, pp. 9-26, 2001.

[14] M. Abd-Elhady, S. Zayed, and C. Rindt, "Removal of dust particles from the surface of solar cells and solar collectors using surfactants," in International Conference on Heat Exchanger Fouling and Cleaning, 2011, vol. 5, no. 10 .

[15] M. B. Lyles, "Medical geology: Dust exposure and potential health risks in the Middle East," in International Seminar On Nuclear War And Planetary Emergencies-42nd Session, 2010, pp. 497-502.

[16] B. Guo, W. Javed, B. Figgis, and T. Mirza, "Effect of dust and weather conditions on photovoltaic performance in Doha, Qatar," in 2015 First Workshop on Smart Grid and Renewable Energy (SGRE), 2015, pp. $1-6$.

[17] D. J. Quesnel et al., "Aspects of particle adhesion and removal," in Developments in Surface Contamination and Cleaning, Elsevier, 2016, pp. 119-145.

[18] C. Borri, M. Gagliardi, and M. Paggi, "Fatigue crack growth in Silicon solar cells and hysteretic behaviour of busbars," Solar Energy Materials and Solar Cells, vol. 181, pp. 21-29, 2018.

[19] C. Bohm, T. Hauck, W. Muller, and A. Juritza, "Probability of silicon fracture in molded packages [ICs]," in 5th International Conference on Thermal and Mechanical Simulation and Experiments in Microelectronics and Microsystems, 2004. EuroSimE 2004. Proceedings of the, 2004, pp. 75-81.

[20] J. Chang and J. Chou, "Fracture strength of tempered glass," Journal of Non-Crystalline Solids, vol. 52, no. 1-3, pp. 395-404, 1982. 